from a large prospective cohort, the Million Women Study (MWS), with the nationwide NHS Cervical Screening Programme records.

Methods Using linked NHS Cervical Screening Programme records for women before they were recruited into the MWS study, participants were classified as non-attenders or attenders for routine cervical screening. Logistic regression models were used to calculate odds ratios (OR) and 95\% confidence intervals (CI) of non-attendance versus attendance by deprivation status, smoking history, body mass index (BMI), parity, age at first birth, oral contraceptive (OC) use and menopausal hormone therapy (MHT) use. All analyses were stratified by year of birth and year of recruitment into the MWS and adjusted for other factors, where appropriate.

Results Of 871,732 study participants who were eligible to have been invited for cervical cancer screening, 25,261 were non-attenders and 846,471 were attenders. The odds of being a non-attender were increased with deprivation (OR [95\%CI] 1.46 [1.40-1.53] for most versus least deprived fifth), obesity (1.38 [1.33-1.43] for BMI $\geq 30$ versus $\left.20-25 / \mathrm{kgm}^{2}\right)$ and smoking (1.25 [1.19-1.30] for heavy current smokers versus never smokers). Nulliparous women were much more likely to be non-attenders (5.80 [5.28-6.38]) and women who were younger at their first birth were also less likely to attend (1.24 [1.12-1.37] for $<17$ versus $\geq 25$ years at first birth). By contrast, women who had used OCs or MHT were much less likely to be non-attenders $(0.38[0.37-0.40]$ for $\geq 10$ years versus never OC use and $0.33[0.32-0.35]$ for current versus never MHT use).

Conclusion In this large cohort of women in England, attendance for cervical screening varied considerably, not only by deprivation, as had been reported previously, but also by lifestyle, reproductive and hormonal factors. Non-attendance was associated with obesity, heavy current smoking, nulliparity, giving birth at younger ages, and non-use of OCs or MHT.

\section{OP04 ANTIOXIDANT BIOMARKERS AND RISK OF PROSTATE CANCER DEATH: A COLLABORATIVE ANALYSIS OF INDIVIDUAL PARTICIPANT DATA FROM 13 PROSPECTIVE STUDIES}

A Perez-Cornago*, on behalf of the Endogenous Hormones, Nutritional. Cancer Epidemiology Unit/Nuffield Department of Population Health, University of Oxford, Oxford, UK

\subsection{6/jech-2020-SSMabstracts.4}

Background Antioxidant micronutrients may affect the development and progression of prostate cancer. We conducted a pooled analysis of the associations of the concentrations of several antioxidant biomarkers with risk of prostate cancer death in the Endogenous Hormones, Nutritional Biomarkers and Prostate Cancer Collaborative Group.

Methods Principal investigators of prospective studies provided individual participant data for prostate cancer cases and controls on circulating concentrations of carotenoids, retinol, and tocopherols, and blood and toenail selenium, including a total of 12 biomarkers. Data were available for up to 1196 prostate cancer deaths and 2441 controls from up to 13 studies. Risk by study-specific fifths of each biomarker was estimated using multivariable-adjusted conditional logistic regression in matched case-control sets.
Results Carotenoids, retinol and tocopherols were not significantly related to risk of prostate cancer death. The only statistically significant finding was an inverse association between toenail selenium concentrations and risk of prostate cancer death (OR for the highest compared with the lowest fifth 0.37 , 95\% CI $0.26-0.52$, the median time of follow-up from blood collection to death from prostate cancer for cases was 11.8 years); however, circulating selenium concentrations were not associated with risk (OR for highest fifth 0.94, 0.641.38).

Conclusion Although we found that men with higher concentrations of toenail selenium had a lower risk of dying from prostate cancer, this should be interpreted cautiously due to the inconsistency between the results for toenail and blood measures of selenium.

Acknowledgements We thank the collaborating studies (ATBC, CARET, CLUE I, CLUE II, EPIC-Europe, EPIC-Norfolk, FMC, HPFS, MCCS, MEC, NLCS, PHS, and PLCO) and participants.

On behalf of the Endogenous Hormones, Nutritional Biomarkers and Prostate Cancer Collaborative Group (EHNBPCCG).

\section{Wednesday 9 September}

\section{Food: Industry}

\section{OP05 UNHEALTHY COMMODITIES AND THE ENGLISH PREMIER LEAGUE. MARKETING GAMBLING, SUGARY DRINKS AND ALCOHOL TO A GLOBAL AUDIENCE}

R Ireland*. Institute of Health and Wellbeing, University of Glasgow, Glasgow, UK

\subsection{6/jech-2020-SSMabstracts.5}

Background There is increasing awareness of the impact of corporate policies on health and how the consumption of unhealthy products contributes to the global burden of noncommunicable disease. Public health academics are reviewing the activities of commercial actors that influence the disease burden and comparing the strategies of these unhealthy commodity industries which include the alcohol, food and beverage and gambling industries. The English Premier League (EPL) is considered to be the most viewed sports league internationally and is broadcast to 212 territories; a truly 'global football league'. The league's commodification and huge audience has enabled a rise in commercial activities and an increased income for clubs through their sponsorship and broadcasting arrangements.

Methods Five EPL matches in 2019 (in the 2018/19 and 2019/20 football seasons) were recorded as broadcast on BT Sport and Amazon Prime in the UK. This study quantified visual marketing references to unhealthy products in the broadcasting. All segments of broadcast (including commercial breaks) were manually coded using a content analysis for marketing references to unhealthy products. Coding variables included location (e.g. pitch border and shirt front) and format (e.g. electronic).

Results In these five EPL matches, a mean of $24.46 \%$ of all footage included at least one reference to an unhealthy commodity. However, this varied considerably between matches with a high of $38.97 \%$ at the Newcastle United v 